\title{
Read Annotation Pipeline for High-Throughput Sequencing Data
}

\author{
James Holt ${ }^{*}$ \\ Dept. of Computer Science \\ University of North Carolina \\ Chapel Hill, NC 27599, USA \\ holtjma@cs.unc.edu
}

\author{
Shunping Huang \\ Dept. of Computer Science \\ University of North Carolina \\ Chapel Hill, NC 27599, USA \\ sphuang@cs.unc.edu \\ Wei Wang \\ Dept. of Computer Science \\ University of California \\ Los Angeles, CA 90095, USA \\ weiwang@cs.ucla.edu
}

\author{
Leonard McMillan \\ Dept. of Computer Science \\ University of North Carolina \\ Chapel Hill, NC 27599, USA \\ mcmillan@cs.unc.edu
}

\begin{abstract}
Mapping reads to a reference sequence is a common step when analyzing allele effects in high throughput sequencing data. The choice of reference is critical because its effect on quantitative sequence analysis is non-negligible. Recent studies suggest aligning to a single standard reference sequence, as is common practice, can lead to an underlying bias depending the genetic distances of the target sequences from the reference. To avoid this bias researchers have resorted to using modified reference sequences. Even with this improvement, various limitations and problems remain unsolved, which include reduced mapping ratios, shifts in read mappings, and the selection of which variants to include to remove biases.

To address these issues, we propose a novel and generic multi-alignment pipeline. Our pipeline integrates the genomic variations from known or suspected founders into separate reference sequences and performs alignments to each one. By mapping reads to multiple reference sequences and merging them afterward, we are able to rescue more reads and diminish the bias caused by using a single common reference. Moreover, the genomic origin of each read is determined and annotated during the merging process, providing a better source of information to assess differential expression than simple allele queries at known variant positions. Using RNA-seq of a diallel cross, we compare our pipeline with the single reference pipeline and demonstrate our advantages of more aligned reads and a higher percentage of reads with assigned origins.
\end{abstract}

*These authors contributed equally to this work.

Permission to make digital or hard copies of all or part of this work for personal or classroom use is granted without fee provided that copies are not made or distributed for profit or commercial advantage and that copies bear this notice and the full citation on the first page. To copy otherwise, to republish, to post on servers or to redistribute to lists, requires prior specific permission and/or a fee.

$B C B$ '13, September 22 - 25, 2013, Washington, DC, USA Copyright 2013 ACM 978-1-4503-2434-2/13/09 ...\$15.00.

\section{Categories and Subject Descriptors}

J.3 [Life and Medical Sciences]: Biology and genetics

\section{General Terms}

Experimentation, Performance

\section{Keywords}

Pseudogenomes, Lapels, Suspenders, merging, mapping rate, parental origin

\section{INTRODUCTION}

Many next-generation sequencing analyses rely on an alignment to a reference genome. Generally, this alignment is to a single reference sequence, regardless of the underlying organism's ploidy. When parental haplotypes differ in their similarity to the reference sequence a significant alignment bias can result [4, 15]. This is particularly problematic when read counts are treated as quantitative measures. Therefore, it is important to use proper reference sequences for read mapping since it will inevitably affect the accuracy of alignments in terms of mapping ratio (i.e., the ratio of reads that are mapped) and mapping locations.

The most commonly used approach is to map reads to a standard reference and rely on the error tolerance of the aligner to compensate for the genomic variations of the target sequence. Gregg et al. 5] aligned F1 hybrids from reciprocal crosses between the isogenic mouse strains CAST/EiJ and $\mathrm{C} 57 \mathrm{BL} / 6 \mathrm{~J}$ to the $\mathrm{NCBI} 37 / \mathrm{mm} 9$ mouse genome and transcriptome to study parent-of-origin effects. However, this approach favors reads with reference alleles, and it is worth noting that the mouse reference genome is largely based on C57BL/6J. This results in a systematic bias, called the reference bias. To reduce this reference bias, Degner et al. 4. proposed masking every known polymorphic location in the reference genome with a third allele. This approach reduces the total number of reads aligned because the added masked alleles always introduce mismatches, which all aligners attempt to minimize. In fact, unmapped reads result when the best mapping considered has excessive mismatches. In RNA-seq experiments, this reduction in mapped 
reads leads to underestimation of expression level of genes with variations 18. Several attempts have been made to create a sample-specific reference genome or transcriptome for alignments. Keane et al. 7 aligned reads from F1 cross of $\mathrm{C} 57 \mathrm{BL} / 6 \mathrm{~J} \times \mathrm{DBA} / 2 \mathrm{~J}$ to a C57BL/6J-based reference genome and an approximate $\mathrm{DBA} / 2 \mathrm{~J}$ genome where known DBA/2J variants, primarily Single Nucleotide Polymorphisms (SNPs), were substituted into the reference. Turro et al. [18] proposed a hybrid pipeline that first aligned reads to a reference genome in order to call SNPs, and then realigned the same reads to a customized transcriptome with the discovered SNPs incorporated. Since single-base substitutions do not change genome coordinates, it is straightforward to embed SNPs. However, this method cannot be easily generalized to other frame-shifting variants such as small indels, inversions and copy number variants (CNVs) to which a sequence aligner is more sensitive. Rozowsky et al. 14 proposed AlleleSeq for constructing a modified diploid genome by inserting SNPs and indels into the reference genome and using this diploid genome as the reference during alignment to avoid errors caused by reference bias. While AlleleSeq is similar to our proposed pipeline, it's limited to diploid organisms. Moreover, as shown below, it analyzes differential expression at variant positions, which will become more difficult as the density of variants increases.

After reads are mapped, the relative read counts in specific regions of the sequence are often used to quantify abundance within a genomic region. In DNA sequencing (DNA-seq), local read counts are used to estimate copy-number gain or loss 19, 11. In RNA sequencing (RNA-seq), local read counts are used to quantify gene expression levels and to identify the isoforms expressed 13,18 . In diploid organisms, researchers have been interested in assessing the differential expression levels between parental haplotypes, (i.e., parentof-origin or allele effects). In a typical analysis of differential expression, the read coverage at each known variant position are partitioned according to allele and then used to estimate the imbalance $5,7,14$. Statistical corrections to the read counts are required when the density of local variations allows multiple variants to fall in the same read, or read-pair. Thus, in regions with dense genomic variations, the quantitative use of read counts is complicated both by an inability to align, and a difficulty of establishing the independence of each variant observation.

We propose a new read annotation pipeline that overcomes most of these problems. It uses multiple-alignments and a merging process in an attempt to resolve a given read's origin.

First, we leverage the existing databases of genomic variants to build custom reference genome sequences for all parental haplotypes, each of which is used in an independent alignment procedure. We call these synthetic genomes pseudogenomes. Unlike most previous methods, different types of variations, such as SNPs, indels, and structure variants (SVs), can all be integrated into the pseudogenomes. Since the coordinates in the alignment to pseudogenomes are no longer relative to the reference due to the incorporated indels and SVs, we remap all positions back to the reference coordinate system after alignment. This remapping enables comparisons of the pseudogenome alignments and allows us to utilize existing annotations (i.e. positions of gene exons and functional elements), which are generally based on the reference sequence's coordinates.
In a second stage, we merge alignments to multiple pseudogenomes and assign an origin to every read. Because of the previous multi-alignment process, each read may be aligned to more than one pseudogenome. Even within the same pseudogenome, depending on the settings of an aligner, reads can be mapped to multiple locations. Although discarding reads with multiple mappings is a common practice $4,7,3,12$, their exclusion can lead to additional biases in downstream estimation [18. In the merging stage of our pipeline, we resolve such multiple mappings where possible by keeping the best choice based on several welldefined criteria. Meanwhile, each read is labeled with its most likely origin based on comparing its mappings in multiple pseudogenomes. This label facilitates downstream analyses, since read counts instead of allele counts are used to assess differential expression, which requires less severe independence assumption $\mathbb{1}^{1}$

\section{METHODS}

In this section, we describe our pipeline for annotating multi-parental sequencing data. For the purpose of discussion, we will assume that the data set being analyzed is RNA-seq from a two-founder diallel cross. The diallel produces samples from crossing two isogenic parental genomes. Our pipeline is not limited to analyzing diallels, nor is it limited to RNA-seq analysis, as discussed in Section 4 For comparison purposes, we also consider a second analysis pipeline that employs a single reference genome and attempts to achieve similar annotations. In all fairness, this single-reference pipeline is only an approximation to the front-ends of other published methods. We have deliberately attempted to separate the annotation phase of sequence analysis from subsequent analyses in our pipeline. Our new pipeline consists of multiple alignments that incorporate all known genetic variants into a genomic model followed by annotation and merging. Assessments of the differential expression levels due to parent, allele, or slice variants are considered downstream uses of our annotations. We contrast our multi-alignment-based approach with a representative reference-based pipeline and highlight their major differences.

\subsection{Single Reference Pipeline}

In traditional reference-based alignment pipelines, shortreads from high throughput sequencers are first mapped and genetic variation is considered afterward. There are significant advantages in using a standard reference genome. In addition to supplying a standard coordinate system for comparison between target genomes, reference coordinates anchor nearly all of the genome's functional annotations, such as gene/exon locations, transcription factor binding sites, and notations of common variants. When all samples are aligned to this reference, genomic comparisons are significantly simplified. However, the mappability to the reference genome is reduced if a sample has a large number of variations from the reference. This results in either a reduction in the number of reads mapped and/or an increase in mapping errors. If the number of errors exceeds the aligner's

\footnotetext{
${ }^{1}$ Measures based on the origin labels of a read need only consider the likelihood that reads are from the same transcript, whereas measures based allele counts at a particular genomic position must, in addition, consider the likelihood that nearby variants are from the same read.
} 


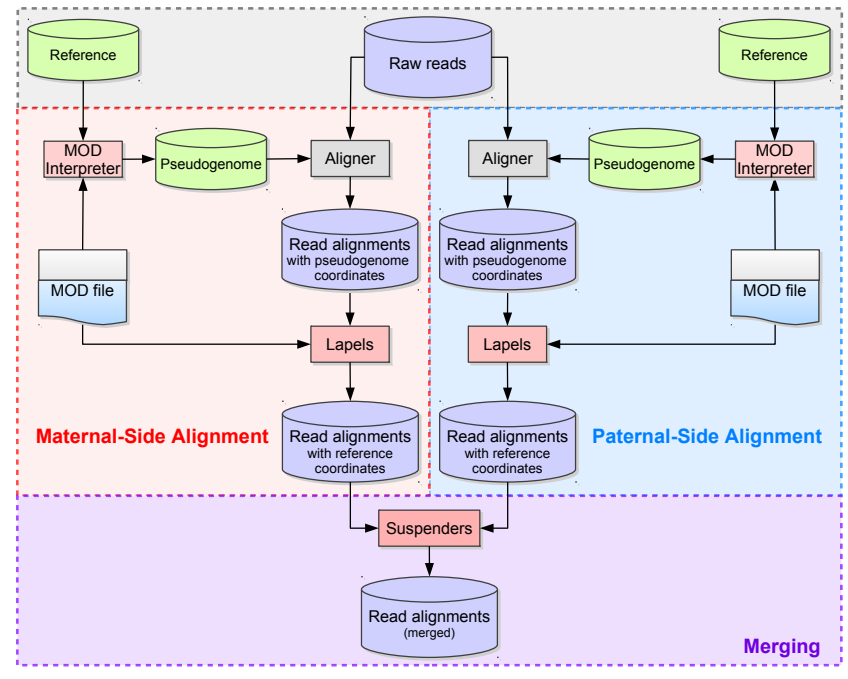

Figure 1: Multi-Alignment Pipeline for a diallel cross. Here we assume that the organisms being considered are diploid. The first step is to create two pseudogenomes using the list of known allelic differences, align the same reads to both pseudogenomes, and convert the mappings back to the reference coordinate system. The details of constructing pseudogenomes and remapping are described in Huang $e t$ al. [6]. Next we merge the two alignments and keep only the best mappings to either pseudogenome in the final merged file.

tolerance, the read will be simply dropped from the output and its information will be lost. In short, a sample that is genetically distant from the reference will typically align fewer reads and with reduced confidence than a sample that is closer to the reference.

There is an extra step of annotating the reads after they are mapped in the alignment. Specifically, the known allelic variations between the parental genomes are used to assign its origin. Consider a diallel cross of two inbred mouse strains as an example. If a mapping shares three SNPs with the maternal strain but only one with the paternal one, the single-reference pipeline assumes that the mapping is from the maternal side. If such counts are the same, suggesting an equal chance of coming from either one, then the strain origin of this mapping cannot be determined.

\subsection{Multi-Alignment Pipeline}

\subsubsection{Pseudogenome Alignment and Annotation}

One of the flaws with the single reference pipeline, applied to an entirely homozygous inbred sample, is the fact that the alignment process does not take into account known allelic differences between the given inbred and the reference. Instead, this is typically handled later during analysis or as a post-alignment annotation (as described in Section 2.1). One of the major differences in our new pipeline is to take advantage of known allelic differences as early in the pipeline as possible. To do this, we first generate a pseudogenome for the inbred based on the reference and known genetic variants (SNPs, insertions, and deletions). This pseudogenome is then used in the alignment process instead of the reference genome, so the end result of alignment is a BAM file 10 using the coordinate system of the pseudogenome.

Separate alignments create problems when comparing samples. If we tried to compare a CAST/EiJ inbred to a $\mathrm{PWK} / \mathrm{PhJ}$ inbred using only the pseudogenome alignments, there would be two different genomic coordinate systems in play. To alleviate this issue, we use the same set of known allelic differences incorporated into the pseudogenome to translate all of the mapped reads back to the reference coordinate system. This involves going through each mapped read and adjusting the mapping position, cigar string, and edit distance to match the reference genome instead of the pseudogenome. This is performed by a Python program called Lapels in our pipeline.

Finally, each mapping is annotated with a series of tags to preserve information from the original pseudogenome alignment. In order to assess the mapping quality, each remapped read retains the cigar string and edit distance from the original pseudogenome mapping as tags. These tags allow us to calculate the original quality scores and preserve information regarding the differences between the reference and pseudogenome mappings for that read.

Unfortunately, alignments are more difficult to assess for multi-parental crosses and diploid organisms in general because there are multiple sets of allelic differences to influence the alignment. In a diallel sample, there are two sets of allelic differences to take into account. We address this problem by constructing pseudogenomes for all contributing founder genomes, performing separate alignments of the full data set to each pseudogenome, and remapping them back to a reference genome while annotating differences as described above (Figure 1).

\subsubsection{Merging - Comparing Alignments}

Next, we consider all annotated alignments as input and merge them into a single output by choosing the best mapping for each read. This is performed by Suspenders in our pipeline. Suspenders sorts the BAM files by read name and systematically compares each alternative mapping. For each read, it extracts all mappings from each Lapels-annotated BAM file before comparing the results. As explained earlier, information from the original pseudogenome alignment is preserved by the annotation step.

The first step in the merge process is to identify the origin of each mapping. To do this, Suspenders first identifies identical mappings based on the mapping start position in the reference genome (chromosome and coordinate), its cigar string in the reference genome, its pairing (either paired or unpaired) flag, its fragment end flag (either first or second), and its quality score (e.g., the Bowtie end-to-end score [1]) from the pseudogenome mappings. The start position and cigar string assure that the two mappings in question cover the same genomic interval in the reference coordinate system. If the pairing and fragment end flags are also the same, it indicates that the read fragment was mapped in the same way in the separate pseudogenome alignments. The final criterion of comparing quality scores implicitly takes allelic differences into account and is discussed in greater detail later. If a read's mappings to two or more pseudogenomes are identical, Suspenders merges the mappings into one logical unit and tags the mapping with a bit vector to identify the origin. For example, for a diallel sample, it would tag 
each mapping with a 2-bit flag set indicating its origin (01: first parent, 10: second parent, 11: either parent). Read mappings that uniquely map to a single pseudogenome are tagged according to their source (i.e. by setting a single bit).

For paired-end reads of a fragment, we "link" parental origins. The main idea is that if one read from a fragment can be assigned to a parent unambiguously, we infer that its mate also came from that same parent, even if the mate contains no informative variants. To illustrate the significance of linking, in our example single-reference pipeline (Section 2.1 , each read is treated independently during the annotation step even if it is mapped as a paired-end read, thus requiring the presence of an informative allele in the read to assign its origin. This approximates the common process of examining alignments only at informative variant positions, while retaining the ability to detect if two variants fall on the same read. In our new pipeline, if two read mappings are properly paired, then they are treated as a single unit throughout the entire merging process. This means that 1 ) the mappings of two properly paired reads will be marked with the same parental origin, 2) the quality score will be calculated once based on the mismatches and indels from the paired mapping, and 3) the merge always prefers a pairedend mapping to one or two single-end mappings from the pair.

\subsubsection{Merging - Filter Pipeline}

Entering into the filtering section of the pipeline, we have a set of possible mappings for a given fragment where each mapping is marked with an origin flag. By sending the set through a series of filters, we remove mappings from the set until only one possible mapping remains for each fragment. Prior to filtering, Suspenders checks to see if any of the possible mappings are mated paired ends. If so, it immediately removes all unpaired mappings from consideration since we prefer a paired mapping over an unpaired one. If there are no paired-end mappings, the mappings are grouped depending upon whether they are the first or second read from the fragment, and a mapping set from each read is independently sent through the Suspenders filters. Note that the two unpaired ends of the same fragment may be filtered in different ways because they are handled separately.

The next step is to send the mapping sets through a series of three filters (shown in Figure 2): Unique, Quality, and Random. If a mapping is output by a filter, we add additional annotation to indicate the chosen mapping as coming from that filter. The Unique filter identifies the reads whose mapping sets contain a single mapping and outputs these mappings. These include all reads having a unique mapping to only a single pseudogenome, and reads mapping to multiple pseudogenomes with single identical mapping as defined previously. In short, the Unique filter outputs simple cases where a read (or two paired reads) has one unique mapping across all pseudogenomes. In Figure 2, reads 1, 2, and 3 each have a single unique positional and score mapping, so they are all output by the Unique filter.

As mentioned earlier, the score comparison is where this pipeline implicitly takes into account allelic variations in the sample. An aligner typically uses a quality score to quantify the mapping quality which is a function of the number of mismatches, insertions, and deletions. Only the mapping(s) with the best score are output. For example, TopHat uses the Bowtie scoring scheme 1] when reporting possible map-

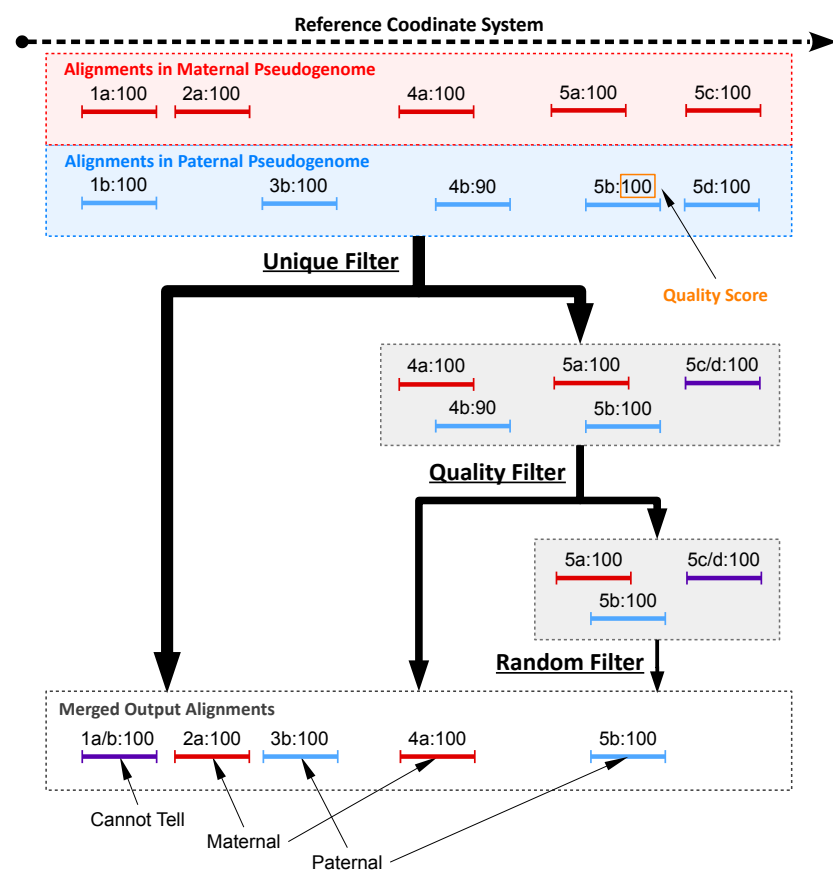

Figure 2: Sample filter path for mapping sets of five reads in a diallel cross labeled such that " $4 \mathrm{~b}: 90 "$ is mapping "b" of read 4 with a score of 90. Prior to filtering, $1 \mathrm{a}$ and $1 \mathrm{~b}$ are combined into $1 \mathrm{a} / \mathrm{b}$ because they have the same position and score in both mappings. Additionally, 5c and 5d are also combined. The mapping sets of reads 1,2 , and 3 are all output by the Unique filter since there is a single positional mapping for each. The mapping sets of reads 4 and 5 have multiple mappings, so they are diverted to the Quality filter by the Unique filter. The Quality filter outputs $4 \mathrm{a}$ since only one mapping of read 4 has the best score (100 compared to 90). The mapping set of read 5 has three mappings with identical scores and is therefore diverted to the Random filter which chooses one mapping arbitrarily. Since there are three in the set, each one has a $33.3 \%$ chance of being chosen, with $5 \mathrm{~b}$ being the arbitrary choice in this example.

pings $8,9,17$. Assume that a read aligns to multiple pseudogenomes that straddles an informative variant caused by a SNP. The mappings to pseudogenomes with the matching variant will have fewer mismatches than that to genomes with the alternate allele. Since sequencing errors are attributes of the read, they contribute mismatches equally to all pseudogenome mappings. In places with no informative alleles, an aligner will report mappings to all genomes with identical number of mismatches. Additionally, if there are multiple variants under a read's mapping, the read may be mapped to multiple positions in the genome, but usually only the best mappings are reported. The Quality filter attempts to simulate this behavior by keeping only the best mappings and their corresponding references. Prior to the filters, identical mappings (with same coordinates and scores) were combined into a single unit. However, the map- 


\begin{tabular}{rrr}
\hline Strain & CAST $\times$ PWK & PWK $\times$ CAST \\
\hline Sample1 & $115,936,064$ & $119,926,340$ \\
Sample2 & $87,988,306$ & $90,706,788$ \\
Sample3 & $142,479,432$ & $170,423,066$ \\
Sample4 & $137,698,560$ & $92,829,168$ \\
Sample5 & $137,953,398$ & $93,801,072$ \\
Total & $622,055,760$ & $567,686,434$ \\
\hline
\end{tabular}

Table 1: Total number of reads for ten samples in two F1 hybrid crosses.

pings were not combined if their scores were different. The Unique filter treats them as two different mappings from distinct origins and passes them to the Quality filter.

Read fragments with multiple mappings (possibly to the same position) are passed to the Quality filter. For each read fragment, we regenerate the original scores of the pseudogenome mappings from the stored cigar strings and edit distances saved during the annotation phase when remapping back to the reference. If only one mapping has the best score, then that mapping is output by the Quality filter. In Figure 2 the mapping set of read 4 has two different mappings (one from maternal and one from paternal). The maternal mapping has a higher quality score, so it is output by the Quality filter.

However, if multiple mappings of a mapping set have the same best score, then we pass those mappings to the Random filter as a last resort. The Random filter will choose only one from the set at random to keep in the merged result. Note that each chosen mapping is tagged with the filter it came from, so the option to remove all mappings from the Random filter can be performed in downstream analysis. In Figure 2 the mapping set of read 5 has three possible mappings with identical scores. Each mapping has a $33.3 \%$ chance of being chosen for the final output. After each read fragment has been processed using the filter pipeline, the final result is a single merged file containing at most one mapping for each single-end read and at most one paired mapping or two unpaired mappings for each pairedend read. Additionally, each read mapping is tagged to identify its pseudogenome origin and the filter that output it during the merge process.

\section{RESULTS}

We evaluated our pipeline using a full diallel cross of two wild-derived inbred mouse strains: CAST/EiJ and $\mathrm{PWK} / \mathrm{PhJ}$. We use the notation of CAST $\times$ PWK to represent the cross whose maternal and paternal parents are $\mathrm{CAST} / \mathrm{EiJ}$ and PWK/PhJ, respectively. Likewise, the reciprocal cross is denoted by PWK $\times$ CAST.

We first extracted mRNA from brain tissues of 10 female samples ( 5 for each cross). Then we used Illumina HiSeq 2000 platform to sequence the transcribed cDNA and obtained around $1.2 \mathrm{G}$ paired-end reads with $100 \mathrm{bp}(2 \mathrm{x} 100)$. The number of reads per sample is shown in Table 1 .

To apply our pipeline, we incorporated SNPs and indels into the mouse reference genome to create strain-specific genomes (pseudogenomes). The variant data was downloaded from the Welcome Trust Sanger Institute 7], while the mouse reference genome sequence was from NCBI MGSCv37.

We used Tophat (v.2.0.5) with default parameters to map

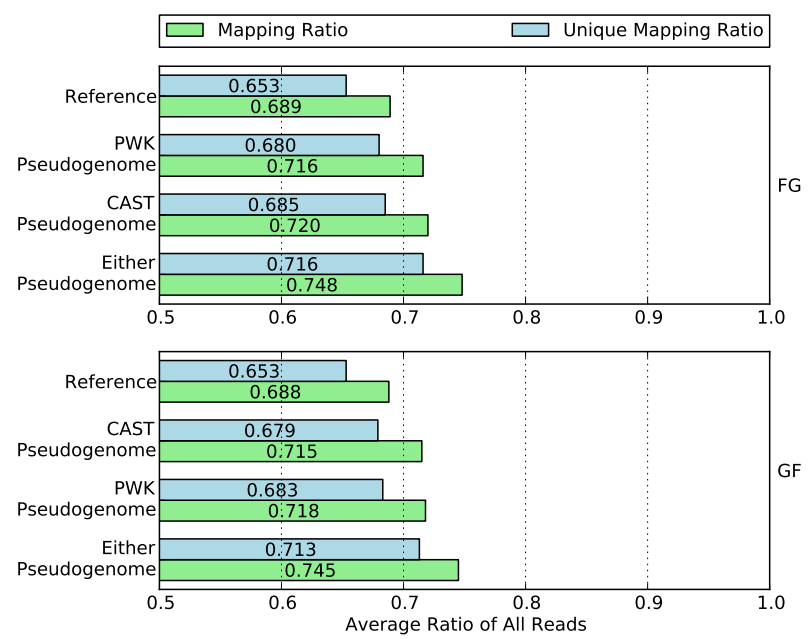

Figure 3: Mapping ratio and unique mapping ratio of reads to the reference genome, two pseudogenomes, and either pseudogenome. Using a single pseudogenome provides a gain of approximately $3 \%$ over the reference genome and using both almost doubles the gain to $6 \%$.

reads to each parental pseudogenome separately. Then, the two sets of alignments were merged as described in Sections 2.2 .2 and 2.2.3 and only one mapping per read was reported in the final output.

\subsection{Comparison of Mapping Ratio}

We examined the fraction of mapped reads from alignments to pseudogenomes, and compared it to the fraction of the same reads when mapped to the standard reference genome. Note that this is an imperfect comparison since, we consider only whether a read maps without considering the accuracy or quality of the mapping. The mapping ratios are shown in Figure 3

Observe that more reads are mapped to each parental pseudogenome than to the reference. The percentage gain is about $3 \%$ for both pseudogenomes in the two crosses. A similar increase can be seen in the percentages of uniquely mapped reads. This suggests that by integrating the variations of parental strains into the reference, we have obtained two better genomes for the reads to align to.

If we consider whether reads mapped to either or both pseudogenomes, the combined recovery rate gain almost doubles to around $6 \%$. To take advantage of this gain, we use the merge process of our method to combine the two sets of alignments in the following section.

\subsection{Comparison of Parental Origin Labeling}

After reads are mapped, our new pipeline and singlereference pipeline next attempt to label the pseudogenome origin of every read where possible. This is a crucial step for downstream analyses which leverage the labels to determine differential gene expression between the parental strains.

In the traditional pipeline, the label is assigned based on the presence of strain-specific alleles seen in the mapped read. For each read mapping, the numbers of maternal al- 

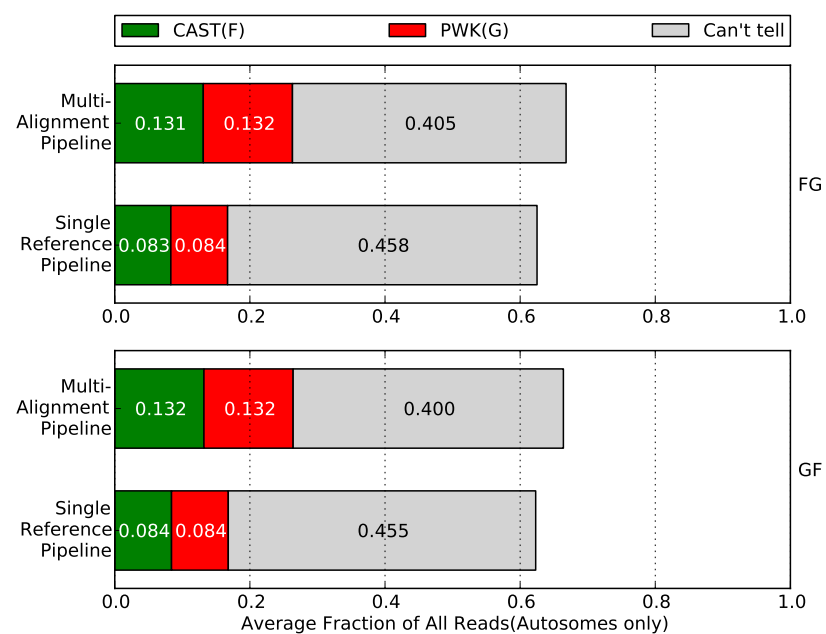

Figure 4: Percentage of parental origin labels in the single reference pipeline compared to the multialignment pipeline. Note that we removed nonautosome mappings, multi-mapped reads, and reads filtered by the Random filter. In general, each strain category gains about $5 \%$ in the multi-alignment pipeline which can be broken down into about $2 \%$ from reads that did not map and about $3 \%$ which were "Can't tell" in the single reference pipeline.

leles and paternal alleles are counted separately and added as a tag to the mapping. If no maternal and paternal alleles are observed or both counts are the same, it will be classified into the "can't tell" category. Otherwise, we chose to label the mapping according to which the allele count was greater.

In our proposed pipeline, the label for each mapping is determined during the merging stage after considering the mappings to multiple pseudogenomes. This process takes place in three filtering stages whose details were discussed in Section 2.2.3

We compared the performance of both pipelines in labeling read origins. To reduce bias between two crosses, we only used reads that mapped to the autosomes. The biases are introduced by mitochondrial RNA expression, which is entirely of maternal origin, and a skewing of the X-inactivation ratios in heterozygotes, which prefers genes expressed from the CAST/EiJ chromosome 2]. Furthermore, reads with multiple mappings are discarded by the single-reference pipeline, which is a common strategy described by many researchers $4,7,3,3,12$. To make the comparison more fair, we ignored the mappings output by the Random filter in our pipeline. Any mappings output by the Random filter are treated as unmapped reads in the figures and tables. The percentage of reads in each category is shown Figure 4.

While only $(4.3 \%)$ more reads are processed in our method than in the traditional one, there is higher percentage of reads assigned to a unique parent of CAST or PWK. Specifically, approximately $5 \%$ of reads are gained for each parental category, while the reads in the "can't tell" class are reduced by over $5 \%$.

To better understand the results, we investigated the re-

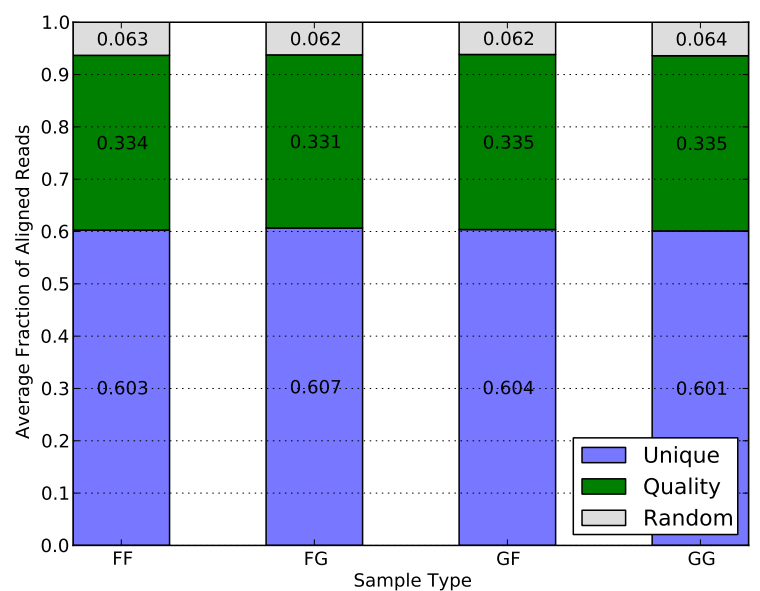

Figure 5: Average filter distribution of mapped reads for diallel samples in the multi-alignment pipeline. F and $G$ denote CAST and PWK respectively. Since each category is approximately equal, it suggests that there is no inherent bias to a strain caused by the filters.

lation between categories in the two pipelines. The results of the two reciprocal crosses are shown in Tables 2 and 3. respectively.

On one hand, a large portion of reads in the CAST category of the single-reference pipeline were assigned to the same category in the multi-alignment pipeline. The percentage is around $96 \%$ for both crosses. The same percentage can be seen in the PWK category as well. This reflects that most reads with non-trivial labels in the traditional singlereference method are covered in the corresponding categories in our approach.

On the other hand, the previous $5 \%$ increase in CAST and PWK categories of our method can be attributed to the $(2 \%)$ reads that cannot be aligned to the standard reference and the $(3 \%)$ reads whose parental origin cannot be determined using the traditional method. This is to be expected since our approach utilizes a merged set of alignments and leverages more information, such as quality score and linking, to decide the origin labels.

\subsection{Performance of Merging}

In order to evaluate the accuracy and consistency of the merging procedure, we applied the same multi-alignment pipeline to inbred samples of CAST and PWK, pretending that they are F1 hybrids crosses (i.e. we performed alignments to both pseudogenomes, annotated reads and remapped them back to the reference, and merged the results). These inbred strains can be considered as the negative controls.

Figure 5 shows the percentage of mapped reads that are output by each of the three filters. Around $60 \%$ of reads are merged in the Unique filter step, suggesting they have either unique mappings in one of the pseudogenomes or identical mappings in both of them. Another $33 \%$ reads have multiple pseudogenome mappings with one mapping better than the 


\begin{tabular}{ccccccc}
\hline & & \multicolumn{4}{c}{ Single Reference Pipeline } \\
& & CAST & PWK & Cannot Tell & Others & Total \\
\hline & CAST & $7.95 \%$ & $0.12 \%$ & $2.89 \%$ & $2.13 \%$ & $13.09 \%$ \\
Multi-Alignment Pipeline & PWK & $0.12 \%$ & $8.03 \%$ & $2.91 \%$ & $2.10 \%$ & $13.16 \%$ \\
& Cannot Tell & $0.03 \%$ & $0.03 \%$ & $39.08 \%$ & $1.33 \%$ & $40.47 \%$ \\
& Others & $0.18 \%$ & $0.19 \%$ & $0.88 \%$ & $32.03 \%$ & $33.28 \%$ \\
& Total & $8.28 \%$ & $8.37 \%$ & $45.76 \%$ & $37.59 \%$ & $100.00 \%$ \\
\hline
\end{tabular}

Table 2: Parental origin of reads from CAST $\times$ PWK samples. Note that non-autosome mappings, multimapped reads, and Random filtered reads are in the "Others" category.

\begin{tabular}{ccccccc}
\hline & & \multicolumn{4}{c}{ Single Reference Pipeline } \\
& & CAST & PWK & Cannot Tell & Others & Total \\
\hline & CAST & $8.09 \%$ & $0.12 \%$ & $2.96 \%$ & $2.05 \%$ & $13.22 \%$ \\
Multi-Alignment Pipeline & PWK & $0.12 \%$ & $8.08 \%$ & $2.95 \%$ & $2.01 \%$ & $13.16 \%$ \\
& Cannot Tell & $0.03 \%$ & $0.03 \%$ & $38.73 \%$ & $1.25 \%$ & $40.04 \%$ \\
& Others & $0.17 \%$ & $0.18 \%$ & $0.87 \%$ & $32.26 \%$ & $33.48 \%$ \\
& Total & $8.41 \%$ & $8.41 \%$ & $45.51 \%$ & $37.57 \%$ & $100.00 \%$ \\
\hline
\end{tabular}

Table 3: Parental origin of reads from PWK $\times$ CAST samples. Note that non-autosome mappings, multimapped reads, and Random filtered reads are in the "Others" category.

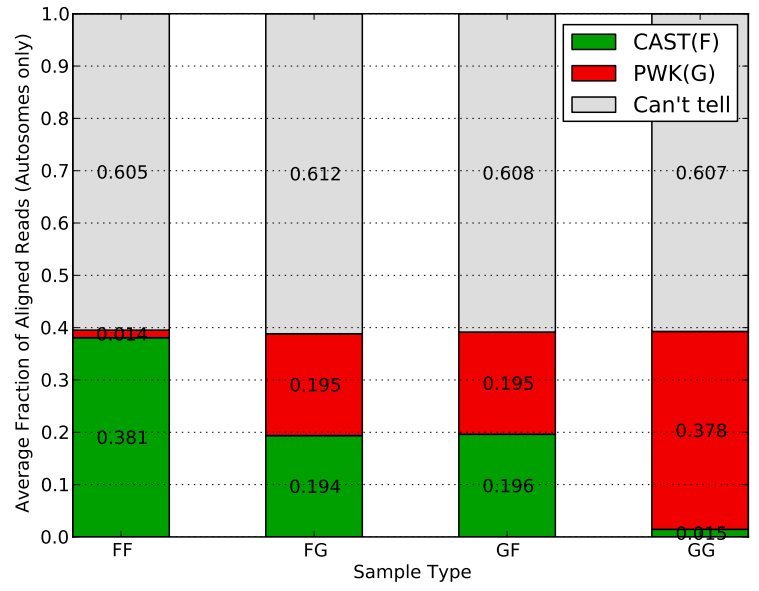

Figure 6: Average parent-of-origin distribution of autosome mapped reads for multiple sample types in the multi-alignment pipeline. In all sample types, approximately $60 \%$ are can't tell with the remaining $40 \%$ divided into parent categories. For inbreds, the vast majority (38\%) are from the associated parent category with only $2 \%$ having a better mapping to the other pseudogenome. For the F1 hybrids, the categories are roughly equal which is expected for the autosomes.

rest, so they are filtered by quality score. The remaining $6 \%$ have multiple mappings with identical quality scores, so one was randomly chosen to be reported in the final output. The consistency of filtering percentages in different strains, including the hybrid crosses and inbreds, suggests that the filters in the merging process do not bias the result.

In Figure 6, we show the percentage of mapped reads in each of the parental origin categories. To avoid the bias caused by the $\mathrm{X}$ chromosome and mitochondria, only reads mapping to autosomes were considered in this analysis. Around $60 \%$ of reads fall into the "Can't tell" class, and this percentage is consistent in F1 hybrids (the second and the third) as well as negative controls (the first and the last). For the residual $40 \%$, we can see the ratio of CAST reads to PWK reads is 1:1 for the F1 hybrids, which is expected because reads are equally likely to come from either parent in autosomes. The ratio for inbred strains, however, is quite different. In fact, the majority of the $40 \%$ are classified to the corresponding inbred strain, while only $1.4 \%-1.5 \%$ are mislabeled. This error rate is likely caused by sequencing noise or unannotated parental alleles.

\section{DISCUSSION AND CONCLUSION}

Although we only applied our pipeline to RNA-seq short reads in this experiment, it is also applicable to other quantitative high-throughput sequence analysis tasks, such as DNA-seq, Chip-seq, DNase-seq, Bis-seq, etc. For example, studies of allele-specific copy number variations can leverage our pipeline for DNA-seq data. The resulting read-origin annotations can be used to estimate the number of DNA copies in different parental haplotypes in later analysis steps.

While we chose to use a diallel experiment to evaluate our new pipeline in Sections 2 and 3 , it is equally applicable to other multi-parental crosses. For example, our multialignment pipeline can be directly applied to Recombinant Inbred Lines (RILs) [16 and back-crosses. For a multiparental cross with $N$ distinct inbred founders, we would generate $N$ pseudogenomes and perform $N$ separate alignments. These alignments can then be merged using $N$ BAM files. In this scenario, each mapping that is saved to the output will have an $N$-bit flag set indicating which files the read was found in. This allows for cases where a mapping's origin is shared/ambiguous between multiple founders. The latest version of Suspenders allows for a variable number of input alignments during the merging process. 
Furthermore, we can incorporate additional filters into the pipeline to better determine the origin of mappings. In our experiment, we only used the Unique and Quality filters as informative filters. This resulted in approximately $5 \%$ of the mapped reads being handled by the Random filter. Adding an additional filters before the Random filter will help to reduce the amount of random choices made in the final output. One possible filter is a Pileup filter based on choosing among otherwise equal mappings the single mapping that has the most surrounding mappings supporting it. To do this, we first find all mapping sets that can be filtered by the Unique or Quality filters and use their chosen mappings to compute the read coverage at each base in the reference genome. Then, any mapping sets that couldn't be resolved using Unique or Quality would compare the pileup coverage of each potential mapping in the set and choose the mapping with the highest coverage. This will be particularly useful for reducing the number of reads that map to pseudogenes in RNA-seq. In cases where the pileups are not significantly different, more computation or simply using the Random filter may be necessary. Suspenders currently has a preliminary version of this filter included in the software package.

To summarize, we propose a new multi-alignment pipeline, which is generic enough to handle reads of various types of organisms from different High-Throughput Sequencing (HTS) techniques. We demonstrated its effectiveness on RNA-seq data from a diallel cross and compared our pipeline with a single-reference pipeline. It is shown that our pipeline outperforms the traditional single-reference based alignment approaches: not only are more reads aligned by our pipeline, but a higher percentage of them are assigned a correct origin.

The two key components of our pipeline, Lapels and Suspenders, are Python scripts which can be downloaded at https://code.google.com/p/lapels/ and https://code. google.com/p/suspenders/

\section{ACKNOWLEDGMENTS}

We thank the members in the UNC CEGS group for strong support to this work. We would also like to thank the three anonymous reviewers for their insightful questions and comments.

This work is supported by NIH P50 MH090338 and NSF IIS-0812464, IIS-1313606.

\section{REFERENCES}

[1] Bowtie2 manual. http://bowtie-bio.sourceforge. net/bowtie2/manual.shtml Accessed: 2013-05-14.

[2] L. H. Chadwick, L. M. Pertz, K. W. Broman, M. S. Bartolomei, and H. F. Willard. Genetic control of x chromosome inactivation in mice: definition of the xce candidate interval. Genetics, 173(4):2103-2110, 2006.

[3] J. S. Cumbie, J. A. Kimbrel, Y. Di, D. W. Schafer, L. J. Wilhelm, S. E. Fox, C. M. Sullivan, A. D. Curzon, J. C. Carrington, T. C. Mockler, et al. Gene-counter: a computational pipeline for the analysis of rna-seq data for gene expression differences. PLoS One, 6(10):e25279, 2011.

[4] J. F. Degner, J. C. Marioni, A. A. Pai, J. K. Pickrell, E. Nkadori, Y. Gilad, and J. K. Pritchard. Effect of read-mapping biases on detecting allele-specific expression from rna-sequencing data. Bioinformatics, 25(24):3207-3212, 2009.
[5] C. Gregg, J. Zhang, B. Weissbourd, S. Luo, G. P. Schroth, D. Haig, and C. Dulac. High-resolution analysis of parent-of-origin allelic expression in the mouse brain. science, 329(5992):643-648, 2010.

[6] S. Huang, C.-Y. Kao, L. McMillan, and W. Wang. Transforming genomes using mod files with applications. In Proceedings of the ACM Conference on Bioinformatics, Computational Biology and Biomedicine. ACM, 2013.

[7] T. Keane, L. Goodstadt, P. Danecek, M. White, K. Wong, B. Yalcin, A. Heger, A. Agam, G. Slater, M. Goodson, et al. Mouse genomic variation and its effect on phenotypes and gene regulation. Nature, 477(7364):289-294, 2011.

[8] B. Langmead and S. L. Salzberg. Fast gapped-read alignment with bowtie 2. Nature methods, 9(4):357-359, 2012.

[9] B. Langmead, C. Trapnell, M. Pop, S. L. Salzberg, et al. Ultrafast and memory-efficient alignment of short dna sequences to the human genome. Genome Biol, 10(3):R25, 2009.

[10] H. Li, B. Handsaker, A. Wysoker, T. Fennell, J. Ruan, N. Homer, G. Marth, G. Abecasis, R. Durbin, et al. The sequence alignment/map format and samtools. Bioinformatics, 25(16):2078-2079, 2009.

[11] A. Magi, L. Tattini, T. Pippucci, F. Torricelli, and M. Benelli. Read count approach for dna copy number variants detection. Bioinformatics, 28(4):470-478, 2012.

[12] V. Missirian, I. Henry, L. Comai, and V. Filkov. Pope: pipeline of parentally-biased expression. In Bioinformatics Research and Applications, pages 177-188. Springer, 2012.

[13] H. Richard, M. H. Schulz, M. Sultan, A. Nürnberger, S. Schrinner, D. Balzereit, E. Dagand, A. Rasche, H. Lehrach, M. Vingron, et al. Prediction of alternative isoforms from exon expression levels in rna-seq experiments. Nucleic acids research, 38(10):e112-e112, 2010.

[14] J. Rozowsky, A. Abyzov, J. Wang, P. Alves, D. Raha, A. Harmanci, J. Leng, R. Bjornson, Y. Kong, N. Kitabayashi, et al. Alleleseq: analysis of allele-specific expression and binding in a network framework. Molecular systems biology, 7(1), 2011.

[15] R. Satya, N. Zavaljevski, and J. Reifman. A new strategy to reduce allelic bias in rna-seq readmapping. Nucleic Acids Research, 40(16):e127-e127, 2012.

[16] L. Silver et al. Mouse genetics: concepts and applications. Oxford University Press, 1995.

[17] C. Trapnell, L. Pachter, and S. L. Salzberg. Tophat: discovering splice junctions with rna-seq. Bioinformatics, 25(9):1105-1111, 2009.

[18] E. Turro, S.-Y. Su, Â. Gonçalves, L. Coin, S. Richardson, A. Lewin, et al. Haplotype and isoform specific expression estimation using multi-mapping rna-seq reads. Genome Biol, 12(2):R13, 2011.

[19] S. Yoon, Z. Xuan, V. Makarov, K. Ye, and J. Sebat. Sensitive and accurate detection of copy number variants using read depth of coverage. Genome research, 19(9):1586-1592, 2009. 